\title{
Application of a-priori hyper-reduction to the nonlinear dynamic finite element simulation of a rolling car tire
}

\author{
Daniel De Gregoriis* \\ Department of Mechanical Engineering \\ KU Leuven \\ Celestijnenlaan $300 \mathrm{~B}$ \\ B-3001, Heverlee, Belgium \\ Email: daniel.degregoriis@kuleuven.be \\ Frank Naets \\ Member of DMMS Lab, Flanders Make \\ Department of Mechanical Engineering \\ KU Leuven \\ Celestijnenlaan $300 \mathrm{~B}$ \\ B-3001, Heverlee, Belgium \\ Email: frank.naets@kuleuven.be \\ Peter Kindt \\ Vibration Mechanics \\ Goodyear Innovation Center* Luxembourg \\ Avenue Gordon Smith \\ L-7750 Colmar-Berg, Luxembourg \\ Email: peter_kindt@goodyear.com \\ Wim Desmet \\ Member of DMMS Lab, Flanders Make \\ Department of Mechanical Engineering \\ KU Leuven \\ Celestijnenlaan $300 \mathrm{~B}$ \\ B-3001, Heverlee, Belgium \\ Email: wim.desmet@kuleuven.be
}

\begin{abstract}
The a-priori Multi-Expansion Modal (MEM) hyper-reduction method for nonlinear structural dynamics finite element problems is described, extended and applied to the dynamic nonlinear finite element simulation of a car tire rolling over a rough road surface, including geometrical, material, follower force and contact nonlinearities. Rather than using time-domain training simulation data, a-priori calculated static nonlinear contact configurations and eigenmodes are used as a reduction basis and to perform the hyper-reduction element sampling. The hyper-reduction element sampling is performed by solving an $L_{1}$ optimization problem subject to a set of equality constraints. This yields a reduced set of elements with an a-priori known cardinality, which depends on the amount of constraints taken into consideration and the reduction basis dimension. It is shown that care has to be taken during the hyper-reduction process when considering distributed contact constraints, as is the case for e.g. a tire
\end{abstract}

${ }^{*}$ Corresponding author 
rolling over a rough road surface. Large speedup factors can be obtained while still retaining a relatively high accuracy, making application of the MEM method suitable to for instance industrial design optimization cases.

\author{
List of Abbreviations \\ ALE Arbitrary Lagrangian Eulerian \\ DOF Degree of Freedom \\ DEIM Discrete Empirical Interpolation Method \\ ECSW Energy Conserving Sampling and Weighting \\ FE $\quad$ Finite Element \\ FBS Force Based Sampling \\ FOM Full Order Model \\ HROM Hyper-Reduced Order Model \\ MOR Model Order Reduction \\ MEM Multi-Expansion Modal \\ NNLS Non-Negative Least Squares \\ OSF Online Speedup Factor \\ POD Proper Orthogonal Decomposition \\ PSD Power Spectral Density \\ ROB Reduced Order Basis \\ ROM Reduced Order Model \\ SBS Stiffness Based Sampling \\ TL Total Lagrangian \\ TSF Total Speedup Factor \\ UDEIM Unassembled Discrete Empirical Interpolation Method
}

\title{
1 Introduction
}

When optimizing the design of a passenger car tire, typically over 50 different performance criteria have to be taken into account. These are related to (but not limited to) e.g. safety, energy efficiency, handling, wear and noise [1]. Due to the complex structure of a typical passenger car tire, most of the criteria are coupled: trying to improve one performance may affect multiple other performances. For example, trying to reduce the airborne exterior noise or structure-borne interior noise by means of increasing the tread thickness and softening the tread material, results in an increased rolling resistance and different handling characteristics [1]. Either an experimental approach or a predictive numerical approach can be used to evaluate the performance of a specific tire design. Due to the costs associated with building and testing tire prototypes, predictive numerical simulations could be considered as a more efficient alternative. In order to cope especially with the increasing need to optimize multiple tire performance criteria simultaneously and have shorter development cycles, the predictive numerical simulation techniques could substitute the time-consuming experiments. Different numerical tire models can be used in a predictive approach, e.g.: (i) Parametric models (e.g. [2, 3]), (ii) Hybrid models (e.g. [4, 5] 6]), (iii) Structural Finite Element (FE) models (e.g. [7,8,9]). While parametric and hybrid models - which can be considered as reduced order models - can be developed for specific boundary conditions and performance assessment, they lack in general the usability and capability to predict the performance of a specific tire design for different criteria. These models also typically need to be tuned in order to match the properties of a specific physical tire, making them not usable for predictive design exploration. Therefore, the FE models (which can be classified as high-fidelity models) typically lend themselves better for this task. However, as these models are based on physics, they describe the physical tire in full detail. As a result, these models are generally complex nonlinear structural FE models ( [7, 8]). Therefore performing predictive numerical simulations using these models often becomes even more time consuming than running the real-life counterpart experiments with built-to-spec prototype tires. As a result, in practice the high-fidelity FE models currently offer no alternative to the experimental approach.

In order to alleviate the computational cost associated with the use of the high-fidelity FE models, the principle of projection-based nonlinear model order reduction (MOR) [10] can be applied. The original full order model (FOM) is transformed into a smaller, lower dimensional reduced order model (ROM) by means of projecting the full order solution space onto a lower dimensional subspace (i.e. the Galerkin method). As demonstrated in e.g. [11] and [12], significant speed-ups can be achieved using nonlinear MOR, therefore re-enabling the use of highly complex high-fidelity FE models. The choice of the subspace basis determines how good the FOM solution is approximated by the ROM solution. Several approaches have been suggested to generate an adequate subspace basis for nonlinear structural dynamics applications. A non-exhaustive overview is given below:

Proper Orthogonal Decomposition (POD) applied to a series of dynamic training snapshots: theoretical and implementation details and examples can be found in e.g. [10, 12, 13] 
Modal Derivatives in combination with e.g. eigenmodes or Krylov modes, e.g. [14, 15, 16 17]

Linear or nonlinear static configurations in combination with e.g. eigenmodes, e.g. [11, 18, 19, 20, 21, 22]

While the POD approach can offer a very efficient low-dimensional subspace basis, relevant simulation training data is required. As will be discussed below, training data can be too expensive to compute, especially when applied in an a-priori method. This is the case for the tire/road problem discussed in this work. The Modal Derivative approach has been shown to be usable for structural dynamics problems with mild nonlinear behavior of the internal force term [15.16 17]. However, highly nonlinear behavior occurs in the tire/road problem, due to the constitutive behavior of the rubber compounds and reinforcements, as well as the contact forces. Therefore, a subspace basis is proposed in this work consisting of static contact configurations and eigenmodes. Due to the configuration dependency of the nonlinear force terms, all of the elements need to be evaluated, assembled and projected again per iteration step. Therefore no real computational cost reduction is achieved by applying MOR.

Recently however, so-called hyper-reduction methods [12, 17, 20,23] have been proposed. These hyper-reduction methods are a class of projection-based nonlinear MOR methods designed specifically for the reduction of nonlinear structural FE models, yielding hyper-reduced order models (HROMs). The projection of the FOM onto a subspace is complemented by a second-tier approximation, which is a necessary step to effectively reduce the computational complexity of the full order FE model [12]. The Energy Conserving Sampling and Weighting (ECSW) method proposed by Farhat et al. [12] has been shown to preserve the structure, symmetry and stability properties of the FOM FE model [13]. Other hyper-reduction methods like the Discrete Empirical Interpolation Method (DEIM) [24] and the Unassembled DEIM method (UDEIM) [25] do not have these necessary properties [13]. The ECSW method [12] requires expensive time-domain training simulations, which have to be performed sequentially as each consecutive timestep depends on the previous one. For large FOMs, as used in tire design optimization problems, this time-domain training approach is too expensive to use, as per design change the time-domain training has to be repeated. The training phase could even be redundant; the training simulations themselves already yield the results necessary to assess the performance of a specific design, and constructing and solving the HROM is therefore not necessary anymore. Thus, there is a clear need for an a-priori method which does not need time-domain training data. Therefore, the application and extension of the a-priori Multi-Expansion Modal (MEM) hyper-reduction method by Naets et al. [20], is proposed in this work. It uses a-priori static training simulations to perform the hyper-reduction, similar to e.g. the static ECSW training method proposed by Rutzmoser and Rixen. [26]. These a-priori static training simulations can be performed in parallel, therefore making the hyper-reduction method suitable even for predictive simulations in tire design optimization problems.

In this work, the proposed hyper-reduction method is applied to a highly nonlinear FE tire model rolling with a constant angular velocity over a rough road surface. Apart from the geometrical nonlinearities as considered by Naets et al. [20], material, follower force and contact nonlinearities are considered in this work as well. The predicted vertical contact forces and tire response due to the rolling are of interest for the assessment of e.g. the structure-borne interior vehicle noise performance of the considered tire design. Prediction of this performance is valuable for e.g. design space exploration. For the rough road surface considered in this work, the tread pattern has a limited influence on the structure-borne noise [27] and therefore only slick (smooth) and ribbed slick tires are considered. As discussed in section 2, a specific formulation is used to model the constant angular velocity rolling of the tire, which assumes the tire to have a constant cross-section. As a result, tread pattern effects cannot be handled in the presented approach. The rough road surface properties are exploited in order to setup the reduction subspace, which is discussed in more detail in section 3 Therefore, specific simulations of rolling over e.g. single obstacles are not in scope of the presented approach. Throughout this work, the rolling of the tire over the rough road surface with a constant angular velocity is referred to as the tire/road problem.

The paper is structured as follows: the specific equations related to the tire/road problem and corresponding practical issues are discussed in section 2 . Construction and properties of the ROB as used in this work is given in section 3 Discussion of suitable hyper-reduction methods is given in section 4 . Application of the proposed hyper-reduction method to the tire/road problem and discussion of related concerns is provided in section 5. Numerical validation and assessment of the proposed hyper-reduction method is given in section 6 . Finally, a conclusion is given in section 7

\section{Equations of motion of the tire/road problem}

The full order model (FOM) of the tire/road problem is described by the semi-discrete nonlinear equations of motion as:

$$
\mathbf{M} \ddot{\mathbf{x}}+\mathbf{G}_{\mathrm{ALE}}(\mathbf{x}, \omega) \dot{\mathbf{x}}+\mathbf{f}(\mathbf{x}, \dot{\mathbf{x}})-\mathbf{f}_{\mathrm{ALE}}(\mathbf{x}, \omega)=\mathbf{f}_{\mathbf{p}}\left(\mathbf{x}, p_{a}\right)+\mathbf{f}_{\mathbf{c}}\left(\mathbf{x}, \mathbf{x}_{\mathbf{r}}\right)+\mathbf{f}_{\mathbf{e}}
$$

The current configuration is defined as $\mathbf{x}=\mathbf{x}_{\mathbf{0}}+\mathbf{u} \in \mathbb{R}^{n}$, where $\mathbf{x}_{\mathbf{0}}$ is the reference configuration and $\mathbf{u}$ the total displacement at time $t$ with respect to the reference configuration. The first and second derivatives of the current configuration with respect to time are denoted as $\dot{\mathbf{x}}$ and $\ddot{\mathbf{x}}$ respectively. The time-dependency is omitted from notation for clarity. Furthermore, $\mathbf{M} \in \mathbb{R}^{n \times n}$ is 
the configuration-independent mass matrix, $\mathbf{G}_{\mathbf{A L E}}(\mathbf{x}, \omega) \in \mathbb{R}^{n \times n}$ the skew-symmetric Gyroscopic matrix, $\omega \in \mathbb{R}^{3}$ the constant tire angular velocity, $\mathbf{f}(\mathbf{x}, \dot{\mathbf{x}}) \in \mathbb{R}^{n}$ the internal force vector, $\mathbf{f}_{\mathbf{A L E}}(\mathbf{x}, \omega) \in \mathbb{R}^{n}$ the rotational inertia force vector, $\mathbf{f}_{\mathbf{p}}\left(\mathbf{x}, p_{a}\right) \in \mathbb{R}^{n}$ the air-pressure force vector and $p_{a}$ the applied tire inflation pressure, $\mathbf{f}_{\mathbf{c}}\left(\mathbf{x}, \mathbf{x}_{\mathbf{r}}\right) \in \mathbb{R}^{n}$ the tire/road contact force vector, $\mathbf{x}_{\mathbf{r}}$ the current road surface and $\mathbf{f}_{\mathbf{e}} \in \mathbb{R}^{n}$ the configuration-independent external force vector. The specific form and structure of the different force terms is discussed in the section below. The tire domain is denoted as $\Omega$ and is discretized by a set of finite elements $E$. The inner surface of the tire, upon which the air cavity pressure acts (the so-called inner liner), is denoted as $\Omega_{p}$ and is discretized by a set of finite elements $E_{p} \subset E$. Air pressure follower forces are applied to part of its boundary, denoted by $\partial \Omega_{p}$. The tire/road contact area subdomain is denoted as $\Omega_{c}$ and is discretized by a set of finite elements $E_{c} \subset E$. Contact forces are applied to part of its boundary, denoted by $\partial \Omega_{c}$. As an ALE-formulation [28] is used to describe the constant angular velocity rotational dynamics, the tire domain does not rotate and the contact subdomain $\Omega_{c}$ does not change with respect to $\Omega$. A schematic overview of the entire domain $\Omega$ and the subdomains $\Omega_{p}, \Omega_{c} \in \Omega$, as well as their respective boundaries $\partial \Omega_{p}$ and $\partial \Omega_{c}$, is shown in Figure 1 . An example of the discretized domain $\Omega$ as used in this work, using a set of elements $E$, is shown in Figure 2.
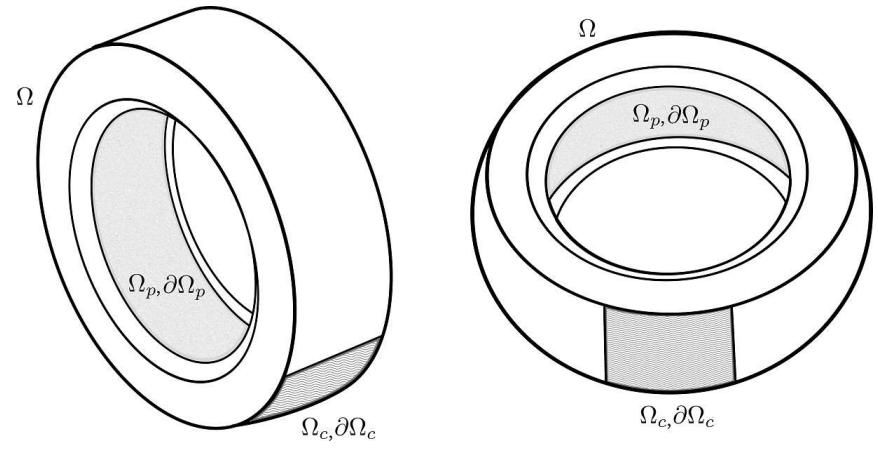

Fig. 1: Tire domain $\Omega$ and subdomains $\Omega_{p}$ and $\Omega_{c}$, with their respective boundaries $\partial \Omega_{p}$ and $\partial \Omega_{c}$.

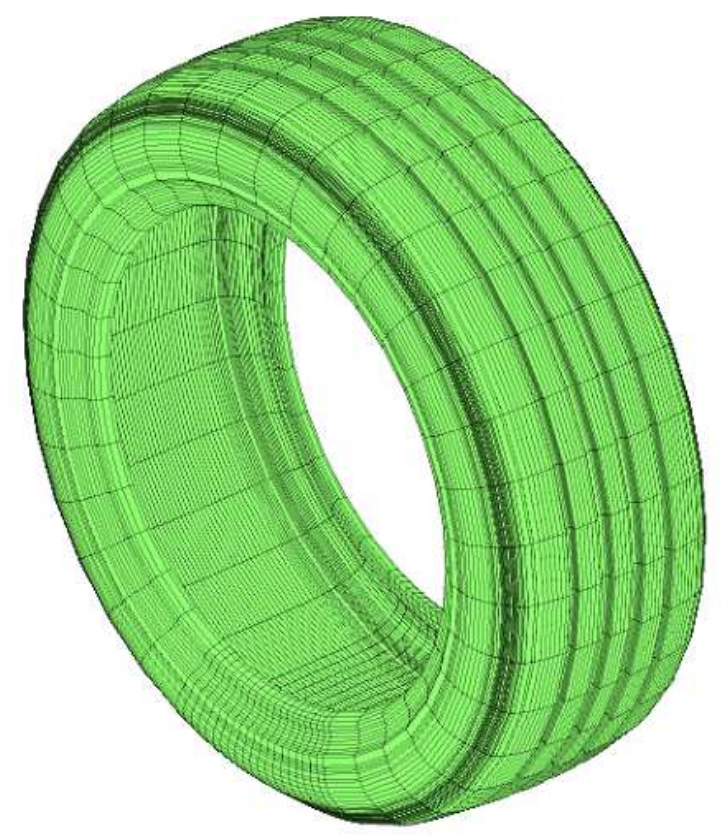

Fig. 2: Discretized tire domain $\Omega$ as used in this work, using an element set $E$ (shown in green). 


\section{Nonlinear internal forces}

In order to accurately model the complex structure of a tire, a general nonlinear strain-displacement relationship and a large set of different nonlinear constitutive models need to be adopted in the high-fidelity numerical tire models. An example of a numerical tire model cross-section, showcasing different subdomains with varying properties, is shown in Figure 3. While all of these subdomains have different constitutive models with varying material parameters, some of the subdomains also incorporate the effects of reinforcement fibers and/or display very large strains (e.g. near the tire/road contact area and tire sidewall). This results in a very heterogeneous set of subdomain properties.

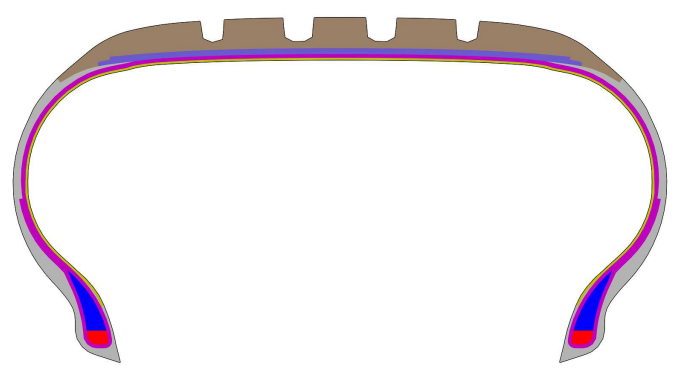

Fig. 3: Example tire cross section domain showcasing different subdomains with different structural properties: e.g. reinforcement fibers, hyperelastic and visco-hyperelastic constitutive behavior.

In this work, the so-called Total Lagrangian (TL) formulation is used [29]. This formulation allows arbitrary large displacements and material point (i.e. fiber) rigid body rotations, using the nonlinear Green-Lagrange strain tensor and the right Cauchy-Green deformation tensor. The TL formulation further allows the use of any type of nonlinear constitutive model, which is necessary to describe the rubber material behavior of the different tire components, whereas e.g. a corotational element formulation [30,31] does not allow to do so [31]. Nonlinear hyper-elastic and visco-hyperelastic constitutive models (as discussed e.g. by de Borst et al. [31]) are used to describe the constitutive behavior of the rubber compounds and reinforcements of the tire. Temperature dependency of the constitutive models is not explicitly taken into account. As constant angular velocity rolling is considered, a constant, steady-state material temperature is assumed and the parameters of the constitutive models are extracted at these steady-state operational temperatures of interest. In order to model the near-incompressibility property of the hyper-elastic constitutive models and to avoid the corresponding locking of the finite element mesh [29], a mixed displacement-pressure $(\mathrm{u} / \mathrm{p})$ formulation [31] is used. The incompressibility constraint is enforced on a material point level (i.e. the numerical integration points) by means of the penalty method [31]. In the mixed $\mathrm{u} / \mathrm{p}$ formulation, both displacements and bulk pressures (or volumetric stresses) are used as field variables. Therefore, the discrete solution space consists of both displacement field and bulk pressure field degrees of freedom. Using finite elements, the internal force term can be written as:

$$
\mathbf{f}(\mathbf{x}, \dot{\mathbf{x}})=\sum_{i=1}^{|E|} \mathbf{f}_{i}\left(\mathbf{x}_{i}, \dot{\mathbf{x}}_{i}\right) \in \mathbb{R}^{n}
$$

The assembled internal force denoted as $\mathbf{f}(\mathbf{x}, \dot{\mathbf{x}})$, while the element contributions are denoted as $\mathbf{f}_{i}\left(\mathbf{x}_{i}, \dot{\mathbf{x}}_{i}\right)$. Here $i$ is an index denoting a finite element and its corresponding set degrees of freedom (DOF). When selecting the element shapefunctions for both the displacement and bulk pressure field, care has to be taken in order to meet the inf-sup condition [29]. Due to the different properties of the subdomains, the corresponding finite elements display similar heterogeneous properties as well. In general, this results in a very heterogeneous nonlinear finite element model, unlike other more homogeneous finite element models used for hyper-reduction applications as in e.g. [12, 13, 17, 26]. As mentioned before, the internal force generally depends on both the current configuration $\mathbf{x}$ and its time-derivative $\dot{\mathbf{x}}$. Because of this dependency, regular projection-based reduction of this term is not efficient and the second-tier hyper-reduction approximation step needs to be performed as well.

\section{Arbitrary Lagrangian Eulerian formulation}

When considering a tire rotating or rolling with a constant angular velocity, it appears natural to adopt a Eulerian formulation to describe the constant, steady-state flow of the tire material through a fixed spatial domain. The deformed tire structure acts as this fixed spatial domain. A Lagrangian formulation can then be used to describe the deformation of the fixed spatial domain that bounds the material flow. Combining both the Lagrangian (material) and Eulerian (spatial) description results in the so-called Arbitrary Lagrangian Eulerian (ALE) formulation. The specific ALE formulation for 
steady-state rolling tires has been discussed in great detail by Nackenhorst [28,32] and is adopted in this work. More specifically, the ALE-formulation for steady-state rolling tires can be interpreted as a decomposition of the mapping of the initial configuration onto the current configuration into two sequential mappings [28]: a mapping onto an intermediate rigid body rotated reference configuration (using a Eulerian description), and the mapping from this rotated reference configuration onto the current configuration (using a Lagrangian description). Again, the interested reader is referred to work by Nackenhorst [28] 32] for full details regarding the derivation of the additional force terms, arising due to the ALE-formulation, and their finite element implementation. One of the main benefits of adopting the ALE-formulation for a tire rolling with a constant angular velocity is that the discretized tire domain is fixed in space, i.e. does not rotate/translate, and that the spatial discretization can therefore be refined locally in the contact boundary [28]. This property is exploited to define the subspace basis in section 3 . A disadvantage is that the rolling structure itself needs to be symmetrical with respect to its axis of rotation [28]. This means that for the case of a tire, only slick tires or ribbed slick tires (which have only longitudinal grooves in the tread layer) can be described using the ALE-formulation. As mentioned in section 1 the tread pattern has a limited contribution to the structure-borne noise tire rolling over a rough road [27]. Therefore, a ribbed slick tire is used in this work and the ALE-formulation can be used. The ALE-specific terms can be written using the finite element discretization as:

$$
\begin{gathered}
\mathbf{G}_{\mathrm{ALE}}(\mathbf{x}, \omega)=\sum_{i=1}^{|E|} \mathbf{G}_{\mathrm{ALE}, i}\left(\mathbf{x}_{i}, \omega\right) \in \mathbb{R}^{n \times n} \\
\mathbf{f}_{\mathrm{ALE}}(\mathbf{x}, \omega)=\sum_{i=1}^{|E|} \mathbf{f}_{\mathrm{ALE}, i}\left(\mathbf{x}_{i}, \omega\right) \in \mathbb{R}^{n}
\end{gathered}
$$

Here again $i$ is an index denoting a finite element and its corresponding set degrees of freedom (DOF). As the ALE-terms depend on the current configuration $\mathbf{x}$, the reduction of these terms needs to be complemented by a hyper-reduction step as well.

\section{Tire/road contact forces}

The road surface asperities are the main source of impact-like excitation for a tire rolling over a rough road surface. The asperities come in and out of contact with the tire tread surface and excite the tire dynamically. A dynamic constraint-based contact mechanics approach, i.e. applying time-dependent contact constraints, is used in this work to model the tire/road surface interaction. Following Wriggers [33], a classical penalty method approach is used to impose the contact constraints. As steady-state free rolling is considered, frictionless contact is assumed. Frictional contact can be included in the proposed hyper-reduction method however, where a specific formulation of the frictional forces [32,34] for the ALE-formulation needs to be adopted. Given the scale of the considered contact problem, a constraint formulation for the normal contact is assumed to be accurate enough. More advanced constitutive models could be used to further model the contact in a micromechanical sense at smaller contact interface scales, as discussed e.g. by Wriggers [33]. Following the ALE-formulation idea of describing the flow of tire material in a Eulerian fashion, a similar approach is used to describe the contact constraints imposed by the road surface: the road material is also modeled as flowing through the spatial tire domain. The road surface configurations are assumed to be known a-priori for all time instances $t$ of interest. Furthermore, the possible contact interface $\partial \Omega_{c}$ is known (due to the ALE-approach) and the road surface is assumed to be rigid. This last assumption is based on typical road material being much stiffer than the tire tread material. Therefore, the road surface can be modeled as a set of geometrical constraints active on $\partial \Omega_{c}$. By considering the time-dependent road surface values in any $\bar{x}_{c} \in \partial \Omega_{c}$, the corresponding time-dependent geometrical contact constraints can be calculated a-priori and in parallel (as the road surface is known per time $t$ ), using e.g. a projection-based contact detection algorithm [33]. The well-known master-slave approach discussed in e.g. [33] is used for the finite element implementation of the contact force term. The rigid road surface is chosen to be the master body. As dynamic impact-like contact is considered, the contact conditions [33] are extended by the so-called persistence condition [35]. Following the results of Suwannachit et al. [36], a relatively high numerical damping factor is included in the time integrator to approximate the persistence condition. The finite element discretization of the contact force term is defined as:

$$
\mathbf{f}_{\mathbf{c}}\left(\mathbf{x}, \mathbf{x}_{\mathbf{r}}\right)=\sum_{i=1}^{\left|E_{c}\right|} \mathbf{f}_{\mathbf{c}, i}\left(\mathbf{x}_{i}, \mathbf{x}_{\mathbf{r}, i}\right) \in \mathbb{R}^{n}
$$

Here $E_{c} \subset E$ is the set of elements used to discretize $\Omega_{c}$ (and its boundary $\partial \Omega_{c}$ ) and $\mathbf{x}_{\mathbf{r}, i}$ are the constraints applied to discretized part of $\partial \Omega_{c}$, i.e. the element $E_{c, i} \in E_{c}$. 


\section{Inflation pressure follower forces}

The overall stiffness of an inflated tire depends largely on the air pressure acting on the inner liner surface. In this work, the external configuration dependent forces due to the inflation pressure are modeled using a follower force formulation [37]. The finite element discretization of the pressure force term is defined as:

$$
\mathbf{f}_{\mathbf{p}}\left(\mathbf{x}, p_{a}\right)=\sum_{i=1}^{\left|E_{p}\right|} \mathbf{f}_{\mathbf{p}, i}\left(\mathbf{x}_{i}, p_{a}\right) \in \mathbb{R}^{n}
$$

Where in this case $E_{p} \subset E$ is the set of elements used to discretize $\Omega_{p}$ (and its boundary $\partial \Omega_{p}$ ) and $p_{a}$ is the pressure applied to discretized part of $\partial \Omega_{p}$, i.e. a face of the element $E_{p, i} \in E_{p}$. Here, $p_{a}$ is thus the tire inflation pressure.

\section{External load forces}

In operating condition, the vehicle weight loads the tire onto the road surface. In an experimental setting, an equivalent load is applied to the tire. This external force term is chosen to not be configuration dependent and is kept constant as well. It is denoted as $\mathbf{f}_{\mathbf{e}} \in \mathbb{R}^{n}$. The external load force could also be assumed to be either configuration and/or time dependent, which would require additional steps in the hyper-reduction process, as discussed in section 3

\section{Time discretization and linearization}

A variant of the implicit generalized- $\alpha$ discrete time integration method, proposed by Arnold and Brüls [38], is chosen to time-discretize the equations of motion (1). This variant of the generalized- $\alpha$ method has second-order accuracy for the acceleration field variables. Theory and implementation details can be found in [38]. An implicit rather than an explicit time integration scheme is chosen, as this allows to use larger timesteps. This results in less timesteps to be evaluated but in general typically a larger overall computational cost, as additional terms need to be evaluated. As an implicit scheme is used, the nonlinear equations of motion (1) are consistently linearized in the spatial domain around the current configuration $\mathbf{x}$, yielding additional stiffness terms. This yields the following set of equations:

$$
\mathbf{M} \ddot{\mathbf{x}}+\mathbf{G}_{\mathbf{A L E}} \dot{\mathbf{X}}+\left(\mathbf{K}-\mathbf{K}_{\mathbf{p}}-\mathbf{K}_{\mathbf{c}}-\mathbf{K}_{\mathrm{ALE}}\right) \Delta \mathbf{u}=\mathbf{f}_{\mathbf{p}}+\mathbf{f}_{\mathbf{c}}+\mathbf{f}_{\mathbf{e}}-\left(\mathbf{f}-\mathbf{f}_{\mathrm{ALE}}\right)
$$

Here $\mathbf{K} \in \mathbb{R}^{n \times n}$ is the internal force tangent stiffness matrix, $\mathbf{K}_{\mathbf{p}} \in \mathbb{R}^{n \times n}$ is the pressure load force tangent stiffness matrix, $\mathbf{K}_{\mathbf{c}} \in \mathbb{R}^{n \times n}$ is the contact force tangent stiffness matrix and $\mathbf{K}_{\mathbf{A L E}} \in \mathbb{R}^{n \times n}$ is the ALE inertia matrix [28]. All terms in (7]) depend on time $t$, but again this dependency has been omitted from notation for clarity. Solving the set of linearized equations of motions (77) per iteration step quickly becomes very costly as the amount of degrees of freedom rises. For industrial-sized design problems, e.g. the tire/road problem discussed in this work, the total computational cost becomes too large and the simulations are no longer feasible to use in a product design context. Therefore, MOR and hyper-reduction are applied to expression (7) in order to lower the overall computational cost.

\section{Defining the reduced order basis}

The full-order solution $\mathbf{u} \in \mathbb{R}^{n}$ (yielding the current configuration $\mathbf{x}$ ), residing in the solution manifold defined by (1), is approximated by the reduced-order solution $\mathbf{q} \in \mathbb{R}^{m}$, which resides in a constant subspace of the solution manifold. The subspace is spanned by the columns of $\mathbf{V} \in \mathbb{R}^{n \times m}$, hereafter called the reduced order basis (ROB). As mentioned in section 2 . the use of the ALE-formulation allows to calculate nonlinear static contact configurations corresponding to specific rolling speeds, contact constraints and external loads. Therefore, similar to the ROBs used in e.g. [11, 18, 19, 22], the ROB as used in this work is generated using static configurations $\chi \in \mathbb{R}^{n \times n_{\chi}}$ to capture the nonlinear deformations and eigenmodes $\Psi \in \mathbb{R}^{n \times n_{\Psi}}$ to capture the dynamics, where $n_{\chi}+n_{\Psi}=m$ :

$$
\mathbf{V}=\left[\chi^{1}-\mathbf{x}_{\mathbf{0}} \cdots \chi^{n \chi}-\mathbf{x}_{\mathbf{0}} \mid \Psi^{1} \cdots \Psi^{n \Psi}\right] \in \mathbb{R}^{n \times m}
$$

This ROB can be calculated a-priori and in parallel. A set of road surface constraints $\left[\mathbf{x}_{\mathbf{r}}{ }^{1} \cdots \mathbf{x}_{\mathbf{r}}{ }^{{ }^{n}}\right.$ ], corresponding to different time instances $t_{i}, i=1 \cdots n_{\chi}$, is sampled from the set of a-priori known road surface constraints. Examples of road surface constraints are shown in Figure 4 In this work, an equidistant sampling is used, but alternative sampling methods, such as e.g. a greedy approach could be further explored as well. 


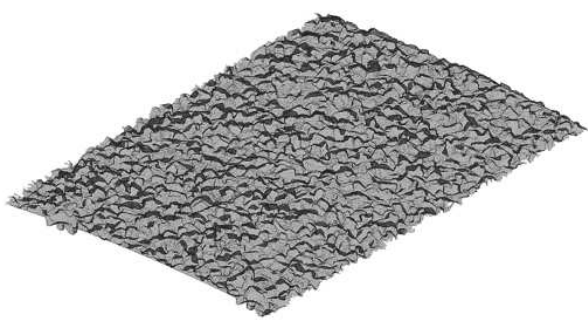

(a)

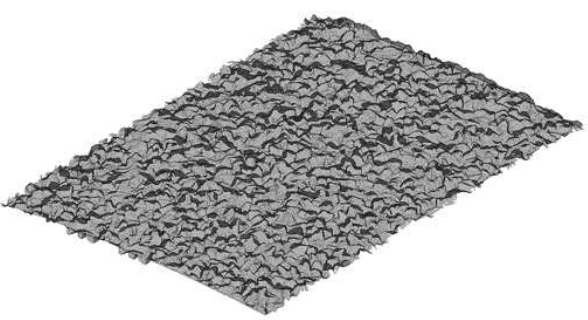

(b)

Fig. 4: Example road surface constraints $\mathbf{x}_{\mathbf{r}}{ }^{l}$, as used for calculating nonlinear static configurations $\chi^{l}$ and during dynamic simulations.

The corresponding static nonlinear contact configurations $\chi^{k}, k=1 \cdots n_{\chi}$ are calculated by solving the following static nonlinear problem:

$$
\mathbf{f}\left(\chi^{k}\right)-\mathbf{f}_{\mathbf{A L E}}\left(\chi^{k}, \omega\right)=\mathbf{f}_{\mathbf{p}}\left(\chi^{k}, p_{a}\right)+\mathbf{f}_{\mathbf{c}}\left(\chi^{k}, \mathbf{x}_{\mathbf{r}}{ }^{k}\right)+\mathbf{f}_{\mathbf{e}}
$$

Note that the ALE force term is included to include the stiffening effect due to the steady-state rotation/rolling. In case a configuration and/or time dependent external force $\mathbf{f}_{\mathbf{e}}$ is considered, static nonlinear contact configurations corresponding to different external force amplitudes should be considered as well, similar to the parametrized ROB as proposed by Blockmans et al. [11]. The static contact configurations can be considered as nonlinear constraint modes, where a distributed set of constraints (the geometrical contact constraints) is applied rather than local unit displacements (as is the case for linear constraint modes [39]). Examples of nonlinear static contact configurations are shown in Figure 5 .

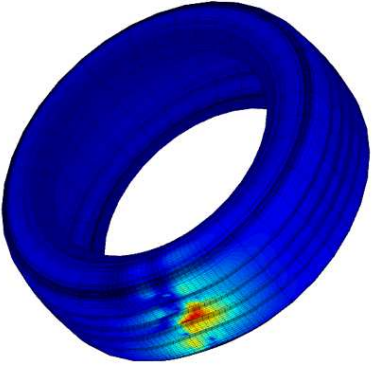

(a)

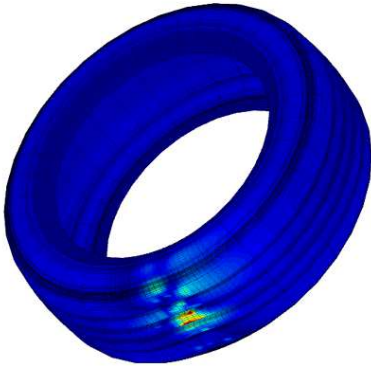

(b)

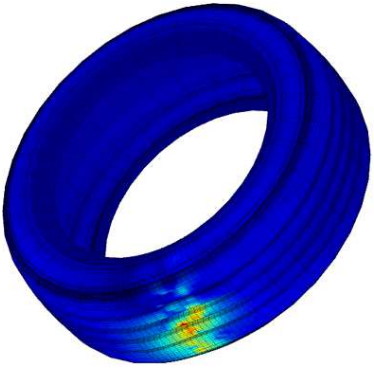

(c)

Fig. 5: Example nonlinear static contact configurations $\chi^{l}$, as used for construction of the ROB.

The amount of nonlinear constraint modes depends on the type of road surface and total amount of rolling distance/time that is simulated. A convergence criterion based on the static projection error can be used to select the minimum amount of nonlinear constraint modes. Given a nonlinear constraint mode $\chi^{*}$ corresponding to a road surface constraint $\mathbf{x}_{\mathbf{r}}{ }^{*} \notin$ $\left[\mathbf{x}_{\mathbf{r}}{ }^{1} \cdots \mathbf{x}_{\mathbf{r}}{ }^{n_{\chi}}\right]$, the static projection error $\varepsilon_{\chi}$ for a static structural reduction basis $\mathbf{V}_{\chi}=\left[\chi^{1}-\mathbf{x}_{\mathbf{0}} \cdots \chi^{n_{\chi}}-\mathbf{x}_{\mathbf{0}}\right]$ can be defined as:

$$
\varepsilon_{\chi}=\frac{\left\|\left(\chi^{*}-\mathbf{x}_{\mathbf{0}}\right)-\mathbf{V}_{\chi}\left(\mathbf{V}_{\chi}^{T} \mathbf{V}_{\chi}\right)^{-1} \mathbf{V}_{\chi}^{T}\left(\chi^{*}-\mathbf{x}_{\mathbf{0}}\right)\right\|_{2}}{\left\|\chi^{*}-\mathbf{x}_{\mathbf{0}}\right\|_{2}}
$$

Here $\|\cdot\|_{2}$ denotes the $L_{2}$-norm. Given then a first choice of $n_{\chi}$ and a user-defined tolerance $\tau_{\chi}$, the number of nonlinear constraint modes can be selected using e.g. Algorithm 1 . If the convergence criterion is not met for a given $n_{\chi}$, the tolerance $\tau_{\chi}$ can either be increased or additional nonlinear constraint modes can be calculated (i.e. increasing $n_{\chi}$ ). The convergence behavior of $\varepsilon_{\chi}$ for the reduced model as used in section 6 is shown in Figure 6 From this figure it is 
clear that a minimal number of nonlinear constraint modes can be selected for which the static projection error converges.

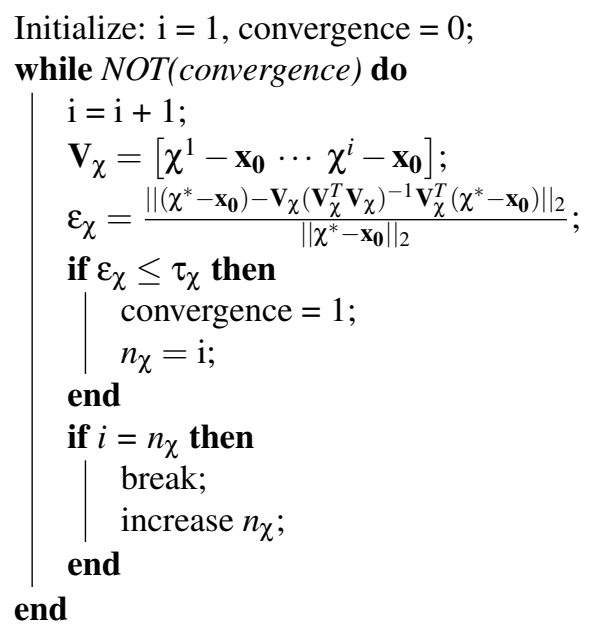

Algorithm 1: Selecting the number of nonlinear constraint modes

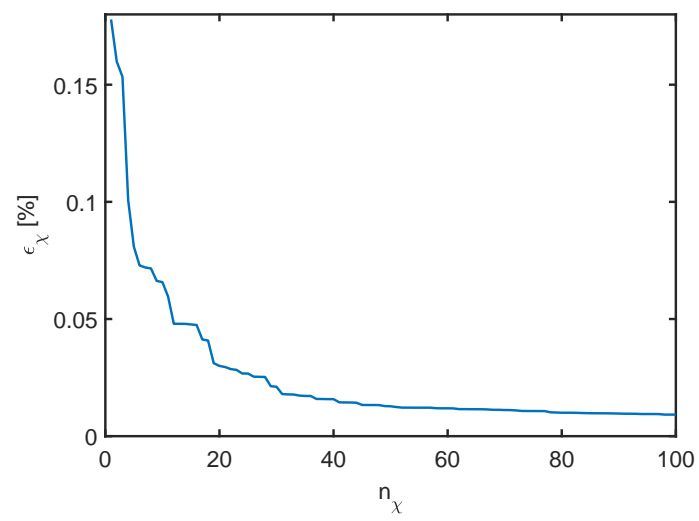

Fig. 6: Convergence behavior of $\varepsilon_{\chi}$ for different values of $n_{\chi}$

Following the MEM method approach as proposed by Naets et al. [20], sets of eigenmodes are calculated around some of the nonlinear constraint modes $\chi^{l} \in \chi, l=1 \cdots n_{l} \leq n_{\chi}$ :

$$
\Psi_{\chi^{l}}=\operatorname{eig}\left(\overline{\overline{\mathbf{K}}}\left(\chi^{l}\right), \mathbf{M}\right) \in \mathbb{R}^{n \times n_{\Psi}}
$$

Here $\overline{\overline{\mathbf{K}}}=\mathbf{K}-\mathbf{K}_{\mathbf{p}}-\mathbf{K}_{\mathbf{c}}-\mathbf{K}_{\mathbf{A L E}}$ is the total tangent stiffness matrix. These sets of eigenmodes are then concatenated, yielding:

$$
\Psi_{\chi}=\left[\Psi_{\chi^{1}} \cdots \Psi_{\chi^{n} l}\right]
$$

And a singular value decomposition is performed on the concatenated eigenspace, where the $n \Psi$ most dominant contributions are kept:

$$
\begin{aligned}
\mathbf{U} \Sigma \mathbf{W}^{T} & =\operatorname{svd}\left(\Psi_{\chi}\right) \\
\Psi & =\mathbf{U}_{i, i=1 \cdots n_{\Psi}}
\end{aligned}
$$

Due to the use of the ALE-formulation, the eigenvalues and eigenvectors vary only slightly with respect to the nonlinear constraint modes $\chi^{l}$. The spread of the first 100 eigenvalues is shown in Figure 7 for four different nonlinear constraint 
modes/nonlinear contact configurations, where it can be observed that the spread indeed is limited. Therefore, the eigenmodes need only to be calculated around a limited set of $\chi^{l}$ to effectively generate a basis that spans the global eigenspace.

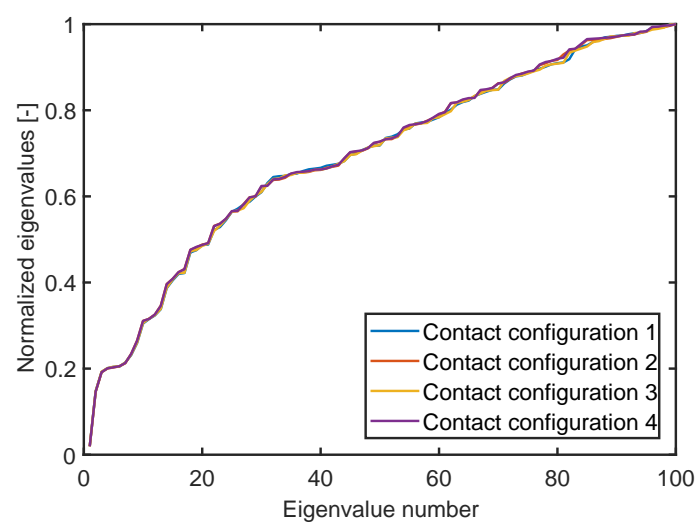

Fig. 7: Normalized eigenvalues corresponding to four different nonlinear static contact configurations $\chi^{l}$.

\section{Hyper-reduction of the tire/road problem}

Following the finite element assembly procedure, the reduced form of the force terms can be written as:

$$
\tilde{\mathbf{f}}(\mathbf{x})=\mathbf{V}^{T} \mathbf{f}(\mathbf{x})=\sum_{i=1}^{|E|} \mathbf{V}_{i}^{T} \mathbf{f}_{i}(\mathbf{x})
$$

Here again $i$ is an index referring to a specific element $E_{i}$ and $\mathbf{V}_{i}^{T}$ and $\mathbf{f}_{i}$ have a sparse structure, only containing the contributions of element $i$. These reduced terms can be approximated further by using the Energy-Conserving Sampling and Weighting (ECSW) hyper-reduction approach as proposed by Farhat et al. [12]:

$$
\tilde{\mathbf{f}}(\mathbf{x}) \approx \overline{\mathbf{f}}(\mathbf{x})=\sum_{i=1}^{\left|E^{s}\right|} s_{i} \mathbf{V}_{i}^{T} \mathbf{f}_{i}(\mathbf{x}) \quad \text { and } \quad s_{i} \geq 0
$$

Here the reduced set of finite elements $E^{s} \subset E$ and their respective nonnegative weights $s_{i}$ are calculated (sampled from the full set $E$ ) using dynamic training data. Given a set of training samples, sampled from a dynamic time-domain simulation: $\mathbf{x}_{i}, i=1 \cdots n_{s}$, the reduced set of elements $E_{s}$ and their respective weights $\mathbf{s}$ are calculated in order to match the projected internal forces. A non-negative least squares (NNLS) problem is solved:

$$
\begin{array}{ll}
\min _{\mathbf{s} \in \mathbb{R}^{|E|}} & \|\overline{\mathbf{H}} \mathbf{s}-\mathbf{b}\|_{2}^{2} \\
\text { subject to } & \mathbf{s} \geq 0 .
\end{array}
$$

Where

$$
\overline{\mathbf{H}}=\left[\begin{array}{ccc}
\mathbf{V}_{1}^{T} \mathbf{f}_{1}\left(\mathbf{x}_{\mathbf{1}}\right) & \cdots & \mathbf{V}_{|E|}^{T} \mathbf{f}_{|E|}\left(\mathbf{x}_{\mathbf{1}}\right) \\
\vdots & \ddots & \vdots \\
\mathbf{V}_{1}^{T} \mathbf{f}_{1}\left(\mathbf{x}_{\mathbf{n}_{\mathbf{s}}}\right) & \cdots & \mathbf{V}_{|E|}^{T} \mathbf{f}_{|E|}\left(\mathbf{x}_{\mathbf{n}_{\mathbf{s}}}\right)
\end{array}\right]
$$

and

$$
\mathbf{b}=\left[\begin{array}{c}
\tilde{\mathbf{f}}\left(\mathbf{x}_{\mathbf{1}}\right) \\
\vdots \\
\tilde{\mathbf{f}}\left(\mathbf{x}_{\mathbf{n}_{\mathbf{s}}}\right)
\end{array}\right]
$$


A sparse NNLS solver, as proposed by Farhat et al. [12], solves the NNLS problem in a suboptimal way, but yields a sparse, effective reduced mesh. As discussed by Farhat et al. [13], other hyper-reduction methods like DEIM [24] and UDEIM [25] do not guarantee stability, nor do they preserve the structure of the finite element assembly procedure. Therefore, the ECSW Hyper-Reduction approach (16) is adopted in this work as well. The cost associated with the dynamic time-domain training simulations and solving the NNLS problem makes the ECSW element sampling method less interesting for an apriori approach. Recently, an alternative a-priori training approach was suggested by Rutzmoser and Rixen [26] that can be combined with the sparse NNLS solver [12]. Since in this work a set of nonlinear constraint modes (due to the contact problem) is used to create the ROB, an alternative element sampling method is used that does not rely on the NNLS approach. More specifically, a variant of the MEM method proposed by Naets et al. [20] is used.

\section{Multi-expansion point modal reduction (MEM) method}

Rather than solving the NNLS problem to perform the ECSW element sampling, the general MEM method element sampling [20] is performed by solving the following $L_{1}$ optimization problem:

$$
\begin{array}{ll}
\min _{\mathbf{s} \in \mathbb{R}^{|E|}} & |\mathbf{s}|_{1} \\
\text { subject to } & \overline{\mathbf{f}}(\mathbf{x})=\tilde{\mathbf{f}}(\mathbf{x}) \\
& \overline{\mathbf{K}}(\mathbf{x})=\tilde{\mathbf{K}}(\mathbf{x}) \\
& \mathbf{s} \geq 0 .
\end{array}
$$

Here $\mathbf{x}$ is the configuration at a specific timestep or loadstep and $E$ is the set of elements evaluated to assemble the force term f. The tangent stiffness matrix is denoted as $\mathbf{K}$. Using the finite element assembly procedure again, the first constraint can be expressed as:

$$
\begin{aligned}
{\left[\mathbf{V}_{1}^{T} \mathbf{f}_{1}(\mathbf{x}) \cdots \mathbf{V}_{|E|}^{T} \mathbf{f}_{|E|}(\mathbf{x})\right] \mathbf{s} } & =\tilde{\mathbf{f}}(\mathbf{x}) \\
\mathbf{A}^{f} \mathbf{S} & =\tilde{\mathbf{f}}(\mathbf{x})
\end{aligned}
$$

For the second constraint, the tangent stiffness matrix $\mathbf{K}$ is vectorized as follows:

$$
\underline{\mathbf{K}}=\left[\begin{array}{c}
\mathbf{K}_{11} \\
\cdots \\
\mathbf{K}_{n 1} \\
\hline \cdots \\
\hline \\
\mathbf{K}_{1|E|} \\
\cdots \\
\mathbf{K}_{n|E|}
\end{array}\right]
$$

The second constraint can then be expressed as:

$$
\begin{array}{r}
{\left[\mathbf{V}_{e, 1}^{T} \mathbf{K}_{e, 1}(\mathbf{x}) \mathbf{V}_{e, 1} \cdots \mathbf{V}_{e,|E|}^{T} \mathbf{K}_{e,|E|}(\mathbf{x}) \mathbf{V}_{e,|E|]}\right] \mathbf{s}=\underline{\tilde{\mathbf{K}}(\mathbf{x})}} \\
\mathbf{A}^{K} \mathbf{S}=\underline{\tilde{\mathbf{K}}}(\mathbf{x})
\end{array}
$$

Solving this problem using an $L_{1}$ optimization algorithm of choice typically leads to a set of elements $E^{s}$ with a cardinality equal to $\frac{m^{2}+m}{2}+m$. Two variants of the general MEM sampling problem can be defined: the stiffness based sampling (SBS) approach and the force based sampling (FBS) approach. The SBS approach uses constraint [25], yielding a set $E$ with cardinality $\frac{m^{2}+m}{2}$. The main benefit of SBS is that only the tangent stiffness matrix corresponding to one configuration needs to be evaluated. However, in case $\frac{m^{2}+m}{2} \geq|E|, E^{s}=E$ and no hyper-reduction is achieved. This disadvantage can arise when a large dimension ROB has to be used. In fact, this issue arises for the tire/road problem discussed in this work, due to the distributed contact problem (resulting in a relatively large set of nonlinear constraint modes) and the relatively large amount 
of eigenmodes necessary to capture the dynamics (which are triggered by the dynamic impact-contact problem). The FBS approach on the other hand considers constraint 22 for multiple configurations $\mathbf{x}_{k}$ :

$$
\begin{array}{ll}
\min _{\mathbf{s} \in \mathbb{R}^{E \mid}} & |\mathbf{S}|_{1} \\
\text { subject to } & \overline{\mathbf{f}}\left(\mathbf{x}_{1}\right)=\tilde{\mathbf{f}}\left(\mathbf{x}_{1}\right) \\
& \vdots \\
& \overline{\mathbf{f}}\left(\mathbf{x}_{k}\right)=\tilde{\mathbf{f}}\left(\mathbf{x}_{k}\right) \\
& \mathbf{S} \geq 0 .
\end{array}
$$

These constraints can be rewritten as:

$$
\begin{array}{r}
\left.\left[\begin{array}{c}
\mathbf{V}_{1}^{T} \mathbf{f}_{1}\left(\mathbf{x}_{1}\right) \\
\cdots \\
\mathbf{V}_{|E|}^{T} \mathbf{f}_{|E|}\left(\mathbf{x}_{1}\right)
\end{array}\right]^{T} \cdots\left[\begin{array}{c}
\mathbf{V}_{1}^{T} \mathbf{f}_{1}\left(\mathbf{x}_{k}\right) \\
\ldots \\
\mathbf{V}_{|E|}^{T} \mathbf{f}_{|E|}\left(\mathbf{x}_{k}\right)
\end{array}\right]^{T}\right] \mathbf{s}=\left[\begin{array}{c}
\tilde{\mathbf{f}}\left(\mathbf{x}_{1}\right) \\
\vdots \\
\tilde{\mathbf{f}}\left(\mathbf{x}_{k}\right)
\end{array}\right] \\
\mathbf{A}_{k}^{f} \mathbf{s}=\left[\begin{array}{c}
\tilde{\mathbf{f}}\left(\mathbf{x}_{1}\right) \\
\vdots \\
\tilde{\mathbf{f}}\left(\mathbf{x}_{k}\right)
\end{array}\right]
\end{array}
$$

While multiple nonlinear force terms corresponding to different configurations $k$ need to be evaluated, solving the FBS MEM problem 26 yields a set $E_{s}$ with a cardinality equal to $k \times m$. Therefore, as long as $k \times m \ll|E|$ and $\frac{m^{2}+m}{2} \geq|E|$, the FBS approach results in a reduced set of elements and can therefore be used for hyper-reduction. Again, an equidistant sampling of static configurations is used, but also here a greedy approach could prove to have some benefits over the equidistant approach.

\section{Constructing the hyper-reduced order model of the tire/road problem}

\section{Kinematics}

Using the ROB as defined in section 3 , the current configuration can be approximated as follows:

$$
\mathbf{x} \approx \mathbf{x}_{\mathbf{0}}+\mathbf{V q}
$$

Where the total displacement $\mathbf{u}$ is thus approximated by $\mathbf{V q}$. Since $\mathbf{V}$ is chosen to be constant, it follows that:

$$
\begin{aligned}
& \dot{\mathbf{x}} \approx \mathbf{V} \dot{\mathbf{q}} \\
& \ddot{\mathbf{x}} \approx \mathbf{V} \ddot{\mathbf{q}}
\end{aligned}
$$

\section{Dynamics}

Inserting approximations (29)- $(31)$ in the linearized equations of motion (77) introduces an error. By using the Galerkin method and thus premultiplying with the transpose of the ROB, $\mathbf{V}^{T}$, this error is eliminated. This is a direct result of the application of the Galerkin method, where the test functions are chosen to be same as the solution functions, yielding an orthogonal error which is thus effectively eliminated after the premultiplication by $\mathbf{V}^{T}$. Further approximation of the configuration dependent terms using the FBS MEM method then yields the hyper-reduced order model (HROM):

$$
\tilde{\mathbf{M}} \ddot{\mathbf{q}}+\overline{\mathbf{G}}_{\mathrm{ALE}} \dot{\mathbf{q}}+\overline{\mathbf{K}} \Delta \mathbf{q}=\overline{\mathbf{f}}
$$

Where

$$
\overline{\mathbf{K}}=\overline{\mathbf{K}}-\overline{\mathbf{K}}_{\mathbf{p}}-\overline{\mathbf{K}}_{\mathbf{c}}-\overline{\mathbf{K}}_{\mathbf{A L E}} \in \mathbb{R}^{m \times m}
$$


and

$$
\overline{\mathbf{f}}=\overline{\mathbf{f}}_{\mathbf{p}}+\overline{\mathbf{f}}_{\mathbf{c}}+\tilde{\mathbf{f}}_{\mathbf{e}}-\left(\overline{\mathbf{f}}-\overline{\mathbf{f}}_{\mathbf{A L E}}\right) \in \mathbb{R}^{m}
$$

As both the mass matrix $\mathbf{M}$ and external force vector $\mathbf{f}_{\mathbf{e}}$ are constant, they are only reduced, yielding:

$$
\tilde{\mathbf{M}}=\mathbf{V}^{T} \mathbf{M V} \in \mathbb{R}^{m \times m}
$$

and

$$
\tilde{\mathbf{f}}_{\mathbf{e}}=\mathbf{V}^{T} \mathbf{f}_{\mathbf{e}} \in \mathbb{R}^{m}
$$

All of the other terms depend on the current configuration $\mathbf{x}$ and are thus hyper-reduced as well. These hyper-reduced terms are discussed below.

\section{Nonlinear internal force and ALE force terms}

In order to evaluate the configuration dependent internal force and ALE force terms, the entire set of elements $E$ needs to be evaluated. Therefore, hyper-reduction is applied to these terms. In order to sample a subset of elements $E_{s}$, the following constraint is used in the FBS MEM problem (26):

$$
\overline{\mathbf{f}}-\overline{\mathbf{f}}_{\mathrm{ALE}}=\tilde{\mathbf{f}}-\tilde{\mathbf{f}}_{\mathrm{ALE}}
$$

Solving the optimization problem 26 then yields the reduced set of elements $E_{s}$ and a set of positive weights $s$. An example subset $E_{S}$ is shown in Figure 8. As expected, more elements are sampled in the contact area (due to the larger deformations and corresponding internal energy) than the top area of the tire. Details of the element selection are shown in Figure 9 , for the tire/road contact area and tire top area respectively.

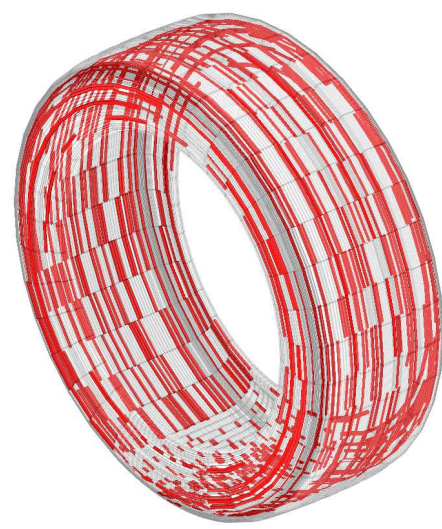

Fig. 8: Example of a set of elements $E^{s}$ (shown in red), sampled from the original set $E$ (shown in white) using a HROM. 


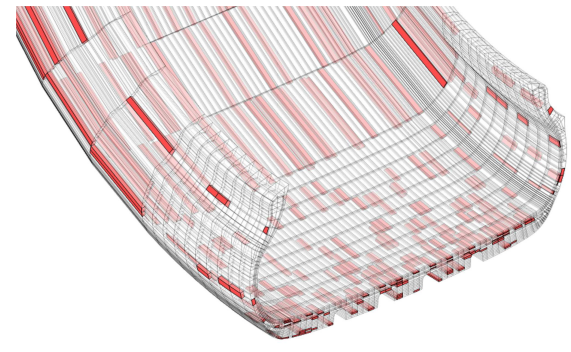

(a)

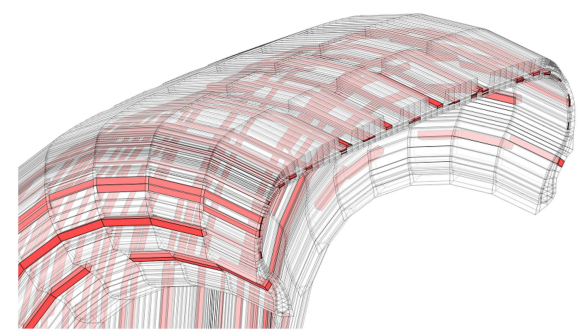

(b)

Fig. 9: Example of a set of elements $E^{s}$ (shown in red), sampled from the original set $E$ (shown in white) using a HROM: detail from the tire/road contact area (a) and detail from the top of the tire (b).

The reduced internal force terms are then approximated via hyper-reduction as:

$$
\begin{gathered}
\overline{\mathbf{f}}=\sum_{i=1}^{\left|E^{s}\right|} s_{i} \mathbf{V}_{i}^{T} \mathbf{f}_{i} \mathbf{V}_{i} \\
\overline{\mathbf{K}}=\sum_{i=1}^{\left|E^{s}\right|} s_{i} \mathbf{V}_{i}^{T} \mathbf{K}_{i} \mathbf{V}_{i}
\end{gathered}
$$

and the ALE force terms as:

$$
\begin{gathered}
\overline{\mathbf{f}}_{\mathbf{A L E}}=\sum_{i=1}^{\left|E^{s}\right|} s_{i} \mathbf{V}_{i}^{T} \mathbf{f}_{\mathbf{A L E}, i} \mathbf{V}_{i} \\
\overline{\mathbf{K}}_{\mathbf{A L E}}=\sum_{i=1}^{\left|E^{s}\right|} s_{i} \mathbf{V}_{i}^{T} \mathbf{K}_{\mathbf{A L E}, i} \mathbf{V}_{i} \\
\overline{\mathbf{G}}_{\mathbf{A L E}}=\sum_{i=1}^{\left|E^{s}\right|} s_{i} \mathbf{V}_{i}^{T} \mathbf{G}_{\mathbf{A L E}, i} \mathbf{V}_{i}
\end{gathered}
$$

\section{Contact forces}

The contact force term is not hyper-reduced, but only reduced. For the specific case of the tire/road problem, the subset of contact elements is small $\left(\left|E_{c}\right| \ll|E|\right)$ and no active contact search has to be performed (the constraints are determined a-priori), as the contact subdomain $\Omega_{c}$ does not change (due to the use of the ALE formulation). Therefore, evaluation of the contact force term has a very low relative computational cost, and no benefit is achieved performing hyper-reduction for this specific application. For more general contact problems, where the contact detection and evaluation phase is far more expensive, hyper-reduction could effectively be used to reduce the associated computational cost. Application of different hyper-reduction methods to more general contact problems is discussed in detail by e.g. Blockmans [40]. Reduction of the contact force term yields:

$$
\tilde{\mathbf{f}}_{\mathbf{c}}=\sum_{i=1}^{\left|E_{c}\right|} \mathbf{V}_{i}^{T} \mathbf{f}_{\mathbf{c}, i} \mathbf{V}_{i}
$$




$$
\tilde{\mathbf{K}}_{\mathbf{c}}=\sum_{i=1}^{\left|E_{c}\right|} \mathbf{V}_{i}^{T} \mathbf{K}_{\mathbf{c}, i} \mathbf{V}_{i}
$$

Additional care has to be taken when evaluating the reduced contact force term. Due to the projection of the FOM on the $\mathrm{ROB}$, the resulting ROM can lock when applying the contact constraints. This is a direct consequence of the reduction of the problem and the choice of the ROB, which is aggravated in the case of the tire/road problem due to:

The heterogeneous nature of the distributed rough road contact problem, where many different constraints are active at the same time. Due to the roughness of the road surface, the local asperities vary greatly in height, often in the $10^{-3} \mathrm{~m}$ range. The tire configuration needs to comply with the road surface to meet the heterogeneous contact constraints locally, requiring large local flexibility which is typically lost after the projection step.

The hyper-elastic, nearly incompressible constitutive behavior in combination with a mixed $\mathrm{u} / \mathrm{p}$ formulation is susceptible to locking [29], even without projection of the FOM on a ROB.

The possible ROM solutions are constrained to lie in the reduction space, and it could be that enforcing the contact constraints results in configurations that do not lie in this space. Therefore, it is suggested to weaken the enforcement of the contact constraints and use a smaller penalty factor $\varepsilon^{*}$ when evaluating the reduced contact force term. A smaller penalty factor allows a larger violation of the contact constraints, and can be seen as (re-)introducing local "flexibility" to prevent the locking of the ROM model. The effect of using a "hard" enforcement of the contact constraints versus a "soft" (relaxed) enforcement is shown in Figure 10, where the relative error on the total vertical contact force $\varepsilon_{f_{c}}$ is compared for a dynamic simulation using the original FOM penalty factor value (hard enforcement) and lower penalty factor (soft enforcement).

An approximate optimal global scaling value of $\varepsilon$, resulting in a smaller penalty factor $\varepsilon^{*}$ (as used for the soft constraint enforcement), can be calculated by means of comparing contact forces corresponding to both the FOM and ROM for a static loading case. The effect of scaling the penalty factor on the the relative error on the total vertical contact force $\varepsilon_{f_{c}}$ is shown in Figure 11 for the case of projecting a static FOM configuration onto the subspace and calculating the corresponding contact force. The same comparison is also made for a dynamic simulation, using different scalings, and is shown in Figure 12 As can be seen from Figure 12, a similar trend can be observed for the dynamic case; the approximate scaling value calculated for the static case can therefore be used as well for the dynamic case. Alternative approaches, optimizing e.g. localized penalty factors, i.e. per spatial set of constraints or per individual constraints, could be explored as well. In this work however, the global approach is used. More pronounced locking effects are expected to occur for lower dimensional subspace bases (as they result in stiffer HROMs); therefore the different approximate optimal scaling values, as calculated for different HROMs, are compared and shown in Figure 13 These HROMS differ in subspace dimension, and their properties are discussed in section 6 It can clearly be observed that decreasing the reduction basis dimension (and corresponding HROM size), results in a decrease of the respective scaling values as well in order to obtain the same degree of relative error. This behavior is to be expected, as more local flexibility needs to be re-introduced for more rigid, lower dimensional subspace bases.

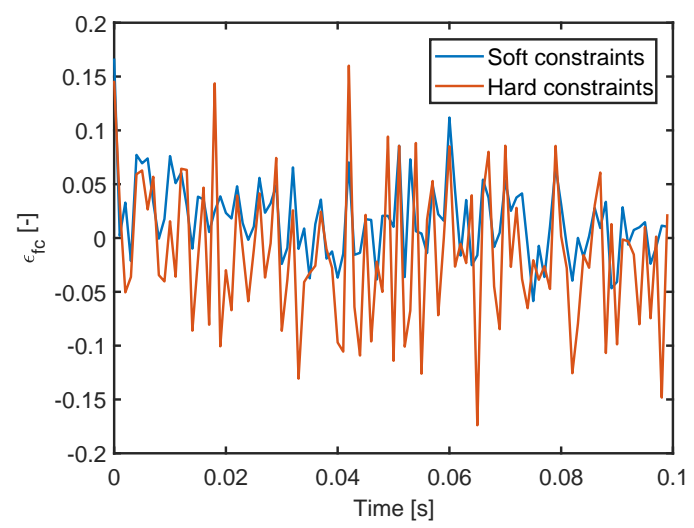

Fig. 10: Relative error on total vertical contact force for a hard and soft enforcement of the contact constraints. 


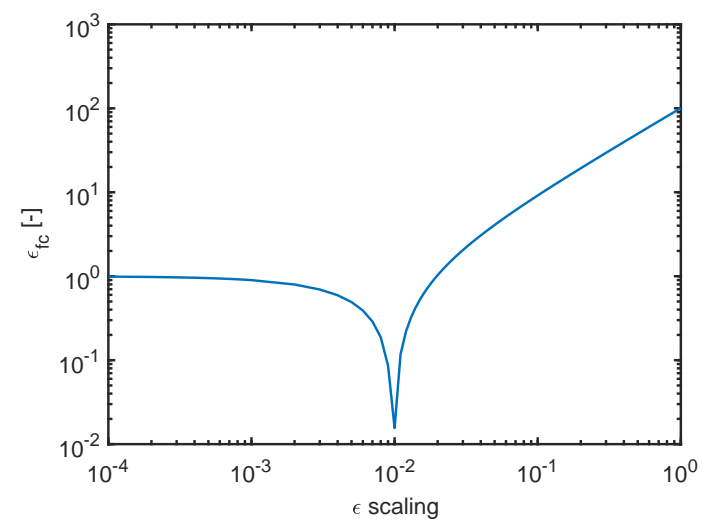

Fig. 11: Relative error on total vertical force for different scalings of the penalty factor $\varepsilon$, for the case of projecting a FOM configuration on the subspace and calculating the corresponding contact forces.

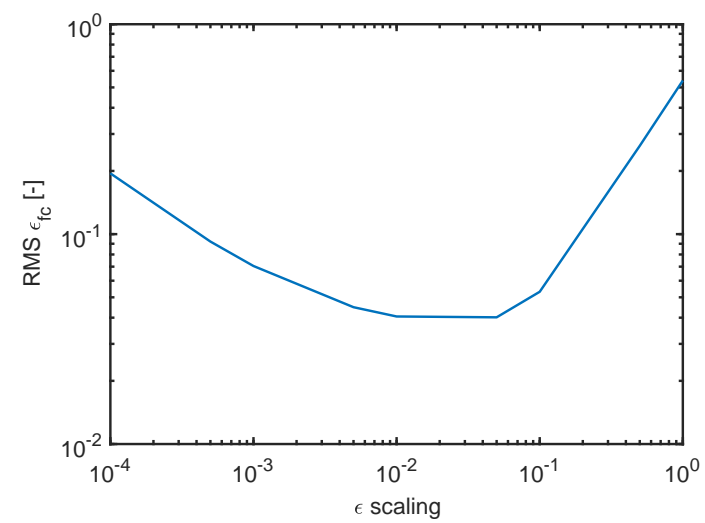

Fig. 12: RMS relative error on total vertical force for different scalings of the penalty factor $\varepsilon$, for the case of dynamic simulations using the different scalings.

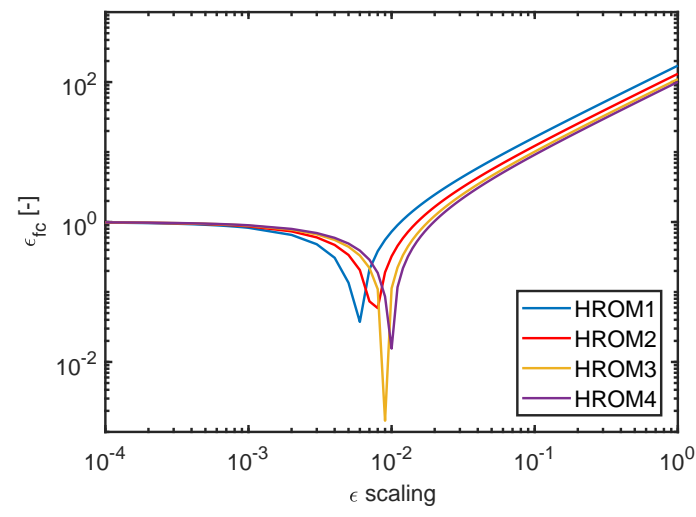

Fig. 13: Relative error on total vertical force for different scalings of the penalty factor $\varepsilon$, for the case of the different HROMs and calculating the corresponding contact forces. 


\section{Pressure force term}

While all of the elements $E$ are used to perform the sampling of the internal and ALE force terms and to assemble their hyper-reduced forms, the subset of elements corresponding to the inner liner domain $\Omega_{p}, E_{p} \subset E$, is used to perform the sampling of the pressure force term, yielding the reduced set of elements $E_{p}^{s}$ and set of positive weights $s$. The following constraint is used in the FBS MEM problem 26:

$$
\overline{\mathbf{f}}_{\mathbf{p}}=\tilde{\mathbf{f}}_{\mathbf{p}}
$$

The pressure force term is then approximated via hyper-reduction as:

$$
\begin{gathered}
\overline{\mathbf{f}}_{\mathbf{p}}=\sum_{i=1}^{\left|E_{p}^{s}\right|} s_{i} \mathbf{V}_{i}^{T} \mathbf{f}_{\mathbf{p}, i} \mathbf{V}_{i} \\
\overline{\mathbf{K}}_{\mathbf{p}}=\sum_{i=1}^{\left|E_{p}^{s}\right|} s_{i} \mathbf{V}_{i}^{T} \mathbf{K}_{\mathbf{p}, i} \mathbf{V}_{i}
\end{gathered}
$$

An example of the reduced set of elements $E_{p}^{s}$ is shown in Figure 14.

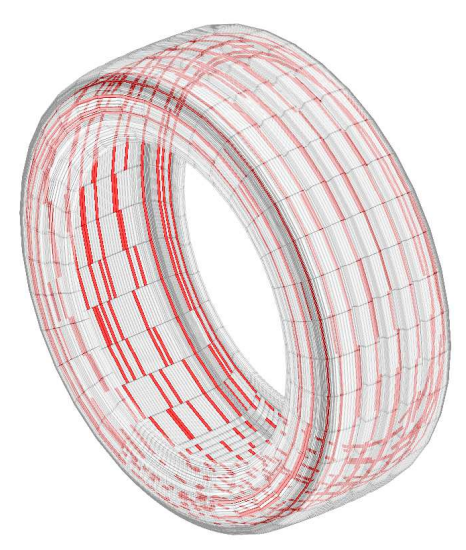

Fig. 14: Example of a set of elements $E_{p}^{s}$ (shown in red), sampled from the original set $E_{p}$ using HROM4 (see section 6 .

\section{Numerical results and validation}

The MEM method is implemented in a nonlinear FE Matlab framework. A fully nonlinear structural FE tire model, as described by equation (1) is used as the FOM. The tire size is 205/55R16 and its geometry is derived from a physical tire design. The FOM consists of over 75,000 elements and 325,000 degrees of freedom. Nonlinear visco-hyperelastic and hyperelastic constitutive models are used to model the rubber compounds and reinforcement materials, similar to the FE tire models described in [41, 42]. All of the constitutive parameters are derived from material sample test data. As discussed before, these properties are assumed to be valid for a constant operational temperature. Sample constitutive parameters and FE tire models (including both the discretized geometry and constitutive models) can be found in e.g. [41 42]. Material testing approaches can be found in the works by e.g. Baranowski et al. [43] and Guo et al. [44]. As discussed by e.g. Vercammen [27] and Behroozi et al. [45], the discretized FOM geometry and constitutive models should match the physical tire geometry and material properties, as the dynamic response of the FOM, and therefore its predictive properties, is highly dependent on these parameters. Therefore, typically a high-fidelity nonlinear FE tire model is required for predictive simulation applications. The tire is mounted on a rigid rim and inflated to the specific air pressure $p_{a}=2.5 \cdot 10^{5} \mathrm{~Pa}$. The numerical test-setup consists of the tire being loaded force-controlled on a rotating drum covered with a rough road surface. The external loading force is set to $4000 \mathrm{~N}$ and held constant. Compliance of the tire fixture assembly is included as a lumped parameter spring/mass assembly. The FOM and a set of road surface contact constraints is shown in Figure 15. 


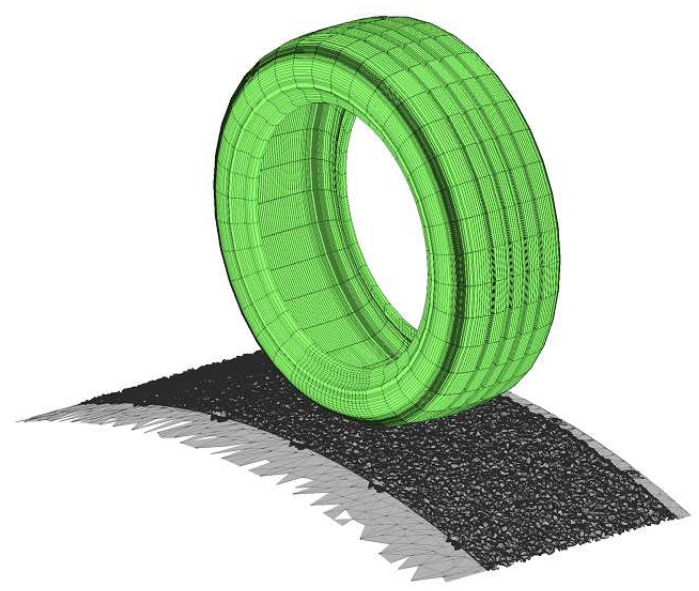

Fig. 15: FOM and set of a road surface contact constraints.

The drum rotates at an angular velocity corresponding to a surface velocity of $13.89 \frac{\mathrm{m}}{\mathrm{s}}$ and drives the rotation of the tire. The tire can thus be considered to be in a free-rolling regime. The applied road surface contact constraints correspond to a maximum excitation frequency of $500 \mathrm{~Hz}$. The corresponding minimal sampling frequency is $1 \mathrm{kHz}$, leading to a minimal timestep $\Delta t=10^{-3}$ s. As discussed before, the hyper-reduced discretized equations of motion 32 are solved using a generalized- $\alpha$ integrator [38]. A smaller timestep is used to ensure stability, as the unconditional stability property does not hold for nonlinear problems [38]. A total of $10^{-1}$ seconds of constant rolling is simulated, using a discrete timestep $\Delta t=10^{-4} \mathrm{~s}$. The relative global error on displacements is defined as

$$
\varepsilon_{x}=\frac{|\mathbf{x}-\tilde{\mathbf{x}}|_{2}}{|\mathbf{x}|_{2}}
$$

and the relative error on the total vertical contact force is defined as

$$
\varepsilon_{f_{c}}=\frac{\left|\mathbf{f}_{\mathbf{c}, \mathbf{z}}-\tilde{\mathbf{f}}_{\mathbf{c}, \mathbf{z}}\right|_{1}}{\left|\mathbf{f}_{\mathbf{c}, \mathbf{z}}\right|_{1}}
$$

where the $L_{1}$ norm is used rather than the $L_{2}$ norm, as the total vertical contact force is of interest. Different HROMS are considered to test and validate the application of the FBS MEM method to the tire/road problem. These HROMS differ in the amount of nonlinear constraint modes and eigenmodes used for ROB construction. The ratio of the number of nonlinear constraint modes $n_{\chi}$ versus number of eigenmodes $n_{\Psi}$ is kept constant for all HROMS. An overview of the different HROMs and their properties is given in Table 1.

Table 1: Definition of the different HROMs

\begin{tabular}{cccc} 
& \# DOFs & $n_{\chi}$ & $n_{\Psi}$ \\
\hline FOM & 326771 & - & - \\
\hline HROM1 & 175 & 25 & 150 \\
\hline HROM2 & 350 & 50 & 300 \\
\hline HROM3 & 525 & 75 & 450 \\
\hline HROM4 & 700 & 100 & 600 \\
\hline
\end{tabular}

\subsection{Influence of the number of configurations used for FBS}

In order to assess the influence of the amount of samples used to calculate the reduced element set $E^{s}$ using the FBS MEM approach, the relative global error on displacements $\varepsilon_{x}$ is compared for different HROMs. These HROMs are con- 
structed using the same reduction basis as HROM4, but using different amounts of samples to create the equality constraints. An overview of the different $\varepsilon_{x}$ values corresponding to the different amounts of samples is shown in Figure 16 As discussed in section 4, equidistant samples are taken from the nonlinear constraint modes. While one sample is not adequate, the use of two samples already provides a good accuracy: including more samples (and thus resulting in a larger HROM) does not yield that much of a better accuracy. When comparing the singular values of $\overline{\mathbf{K}}$, a similar trend can be observed. The first 400 singular values corresponding to one to four samples respectively, are shown in Figure 17 . In case two samples or more are used, the singular values corresponding to the different amount of samples tend to converge to each other. The singular values corresponding to the one sample case do not converge to the other cases, thus showing similar behavior as shown in Figure 16 .

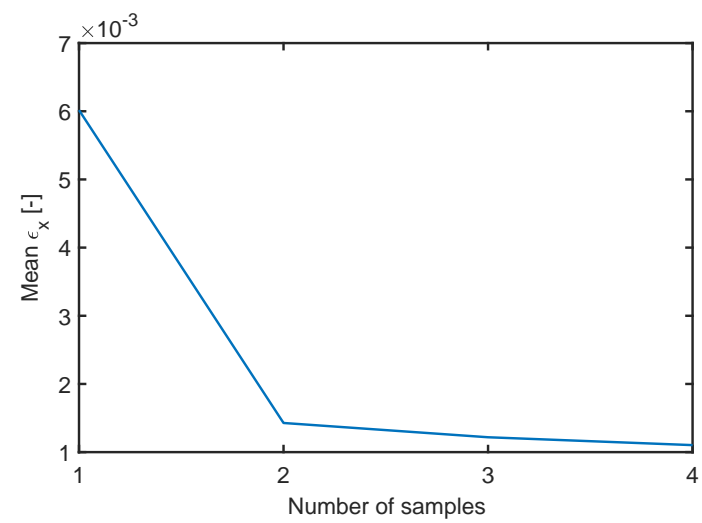

Fig. 16: Mean relative error on total global displacements for different amounts of samples used for the FBS approach.

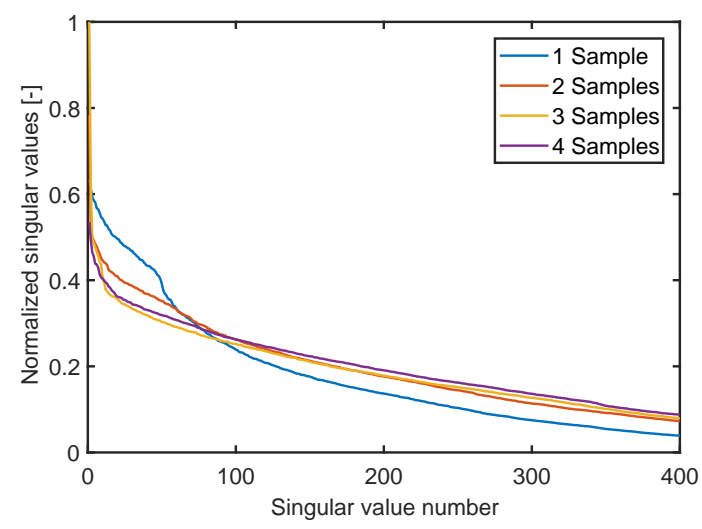

Fig. 17: Normalized singular values of $\overline{\mathbf{K}}$ corresponding to different amounts of samples used for the FBS approach.

\subsection{Global performance}

The global performance of the FBS MEM method is compared for the different HROMs. The same amount of samples is used to calculate $E^{s}$ and $E_{p}^{s}$ for all HROMs. The corresponding approximate optimal static penalty scaling factor is used for each HROM. An overview is given in Table 2. The online speedup factor is denoted as OSF (the time-domain simulation speedup), while the total speedup factor is denoted as TSF. It should be noted that TSF includes a fixed ROB calculation cost, which means that the TSF will tend to the OSF for longer simulated times. The TSF also highly depends on parallelization (to calculate e.g. the road surface constraints and nonlinear static configurations in parallel) and can therefore be optimized even further. In general, applying the FBS MEM method to the tire/road problem allows for good speedups, i.e. from several weeks to hours, while still retaining a relatively high accuracy, as demonstrated by the results shown in Table 2 
Table 2: Global performance of the FBS MEM method for different HROMs

\begin{tabular}{cccccccc} 
& \# DOFs & $|E|$ & $\left|E_{p}\right|$ & $\varepsilon_{x}$ & $\varepsilon_{f_{c}}$ & OSF & TSF \\
\hline FOM & 326771 & 75400 & 6552 & 0 & 0 & 1 & 1 \\
\hline HROM1 & 175 & 350 & 175 & 0.001947 & 0.05719 & 336.56 & 13.81 \\
\hline HROM2 & 350 & 700 & 350 & 0.001599 & 0.04929 & 159.72 & 13.21 \\
\hline HROM3 & 525 & 1050 & 525 & 0.001519 & 0.04947 & 93.54 & 12.48 \\
\hline HROM4 & 700 & 1400 & 700 & 0.001428 & 0.04486 & 59.36 & 11.59 \\
\hline
\end{tabular}

The vertical displacement of a node in the tire/road contact area, undergoing large deformations, is shown in Figure 18 for both the FOM and the HROM4, showcasing the relatively high accuracy of the application of the FBS MEM method to the tire/road problem. Note that due to the use of a smaller penalty factor $\varepsilon$, a larger penetration occurs between the tire and the road surface (larger constraint violation), thus yielding larger local displacement errors at time instances where contact occurs for a specific point in $\partial \Omega_{c}$.

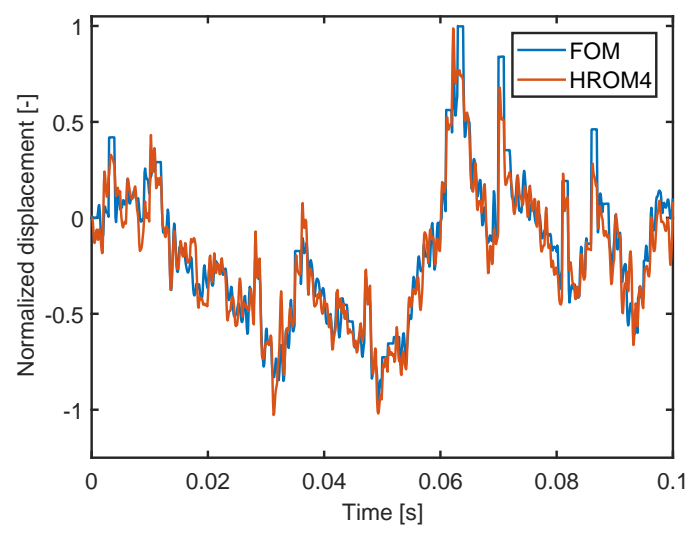

Fig. 18: Normalized vertical displacement of a contact node in the tire/road contact area.

A physical test tire with the same properties as the FOM is experimentally tested using the same boundary conditions as the numerical test setup. The normalized Power Spectral Density (PSD) of the experimental vertical hub force data and the numerical vertical hub force data, calculated using HROM4, is shown and compared in Figure 19. Here, 1s of rolling is considered and several overlapping time windows of $0.5 \mathrm{~s}$ each are used to achieve a frequency resolution of $2 \mathrm{~Hz}$ and eliminate measurement noise. It should be noted that $1 \mathrm{~s}$ of rolling would have taken several months of simulation time using the FOM, whereas the HROM requires only hours of simulation time. As can be seen, a good correspondence between both the experimental and numerical data can be observed. Given the OSFs and TSFs, as well as the overall accuracy of the HROM with respect to the FOM and the accuracy of the HROM with respect to experimental data, the proposed hyperreduced approach could thus be considered as a predictive numerical alternative approach to experimental testing for tire design performance assessment. More specifically, the proposed predictive hyper-reduction approach is approximately ten times faster than an equivalent experimental approach, showcasing the benefit of using the hyper-reduction approach over both the experimental and FOM numerical approaches for tire design performance assessment and e.g. tire design space exploration. As the tire air cavity is not modeled, the air cavity dynamics are not present in the FOM data. However, an additional acoustic model could be included in both the FOM and HROM, as this does not interfere with the proposed hyper-reduction method. 


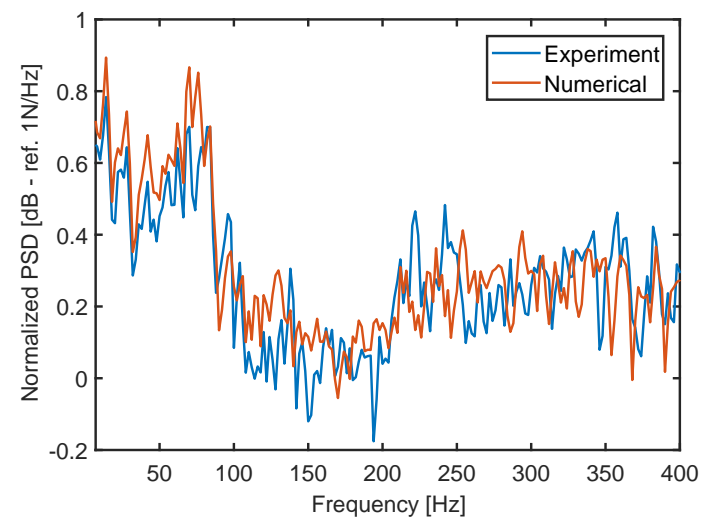

Fig. 19: Normalized PSD comparison between experimental vertical hub force data (shown in blue) and numerical vertical hub force (shown in red), calculated using HROM4.

\section{Conclusion}

The application and extension of the a-priori MEM hyper-reduction method to a nonlinear structural dynamics FE problem containing geometrical, material follower force and contact nonlinearities, such as the tire/road problem, is described in this work. More specifically, the FBS MEM method is chosen to hyper-reduce the tire/road problem, as it allows to perform the a-priori element sampling using nonlinear static contact configurations rather than time-domain training data. The FBS MEM method is a variant of the general MEM method [20], where hyper-reduced and reduced internal forces corresponding to multiple configurations are matched rather than matching the hyper-reduced and reduced internal force and/or tangent stiffness matrix corresponding to one configuration (i.e. the general and SBS MEM approaches, respectively). Due to the matching of internal forces, a smaller reduced set of elements is obtained than the one that is obtained using the general and SBS MEM method. Due to the large dimension of the ROB used to reduce the FOM nonlinear tire model, both the general and SBS MEM method result in a reduced set of elements that is too large to obtain any hyper-reduction. Therefore, the FBS MEM method is chosen as the alternative hyper-reduction method for the tire/road problem.

A constant ROB consisting of nonlinear static (steady-state) contact configurations and eigenmodes is proposed, thereby exploiting the properties of the ALE-formulation used to describe the constant rolling of the tire over the rough road surface. The nonlinear static contact configurations correspond to geometrical rough road surface constraints. These constraints can be calculated a-priori, which allows to calculate the corresponding contact configurations a-priori and in parallel as well. The eigenmodes are calculated around a set of contact configurations. Due to the use of the ALE-formulation, the eigenvalues and corresponding eigenmodes are not too sensitive to the configuration around which they are calculated: this allows to use only a limited set of contact configurations to generate a span of the overall eigenspace. It is shown that care has to be taken when including heterogeneous, distributed contact constraints in the ROM, as locking of the ROM can occur. When using a penalty method to enforce the contact constraints, lowering the penalty factor is shown as a possible way to alleviate this problem. Furthermore, at least two samples need to be used when performing the force based element sampling, as using only one sample leads to larger approximation errors and divergence.

Application of the FBS MEM method to the highly nonlinear tire/road problem shows that large speedups can be achieved while still retaining a relatively high accuracy for the HROM with respect to the FOM. A good accuracy can also be observed when comparing the HROM vertical hub force PSD with the experimental vertical hub force PSD. Application of the proposed hyper-reduction method to the nonlinear FE tire models in this work, as used to predict vertical contact forces and tire response in order to assess the structure-borne interior vehicle noise performance of the considered tire design, results in simulation times approximately ten times faster than the equivalent experimental approach. The reported speedups and accuracy therefore allow to use high-fidelity nonlinear structural FE models, such as the nonlinear tire models used in this work, in a design optimization context. The use of nonlinear static configurations rather than time-domain data to both create the ROB and HROM, is essential in order for the hyper-reduction approach to be usable in a design context. Future research will focus on e.g. the determination of an optimal set of nonlinear constraint modes and eigenmodes for the ROB, optimizing the local scaling of the penalty factors, as well as application of the MEM method to multi-physical problems.

\section{Acknowledgements}

The research of Daniel De Gregoriis is funded by an AFR-PhD grant supported by the Fonds National de la Recherche, Luxembourg (AFR PhD Grant Agreement PhD 2015-1, Project Reference 10222097). The Fund for Scientific Research Flanders (F.W.O.) is gratefully acknowledged for the support of the postdoctoral research of Frank Naets. The Industrial Research Fund KU Leuven is gratefully acknowledged for its support. 


\section{References}

[1] Gent, A. N., and Walter, J. D., 2006. The Pneumatic Tire. University of Akron/ United States. National Highway Traffic Safety Administration.

[2] Gipser, M., 2007. "Ftire - the tire simulation model for all applications related to vehicle dynamics". Vehicle System Dynamics, 45, January, pp. 139-151.

[3] Oertel, C., and Fandre, A., 1999. "Ride comfort simulations and steps towards life time calculations: RMOD-K and ADAMS". In Proceedings of the International ADAMS Users Conference.

[4] Alujevic, N., Campillo-Davo, N., Kindt, P., Desmet, W., Pluymers, B., and Vercammen, S., 2015. "A simplified tire model based on a rotating shell". In 4th International Tyre Colloquium, University of Surrey (Guildford, Surrey, GU2 7XH, United Kingdom).

[5] Kindt, P., Sas, P., and Desmet, W., 2009. "Development and validation of a three-dimensional ring-based structural tyre model". Journal of Sound and Vibration, 326(3), pp. $852-869$.

[6] Larsson, K., and Kropp, W., 2002. "A high-frequency three-dimensional tyre model based on two coupled elastic layers". Journal of Sound and Vibration, 253(4), pp. $889-908$.

[7] Brinkmeier, M., Nackenhorst, U., Petersen, S., and Von Estorff, O., 2008. "A finite element approach for the simulation of tire rolling noise". Journal of Sound and Vibration, 309(1), pp. 20-39.

[8] Gonzalez Diaz, C., Kindt, P., Middelberg, J., Vercammen, S., Thiry, C., Close, R., and Leyssens, J., 2016. "Dynamic behaviour of a rolling tyre: Experimental and numerical analyses". Journal of Sound and Vibration, 364, March, pp. 147-164.

[9] Vercammen, S., Kindt, P., Gonzalez Diaz, C., and Desmet, W., 2014. "Synopsis of the experimental and numerical tire dynamic characterization". In Fisita 2014.

[10] Benner, P., Ohlberger, M., Cohen, A., and Willcox, K., 2017. Model Reduction and Approximation. Society for Industrial and Applied Mathematics, Philadelphia, PA.

[11] Blockmans, B., Tamarozzi, T., Naets, F., and Desmet, W., 2015. "A nonlinear parametric model reduction method for efficient gear contact simulations". International Journal for Numerical Methods in Engineering, 102(5), pp. 1162 1191.

[12] Farhat, C., Avery, P., Chapman, T., and Cortial, J., 2014. "Dimensional reduction of nonlinear finite element dynamic models with finite rotations and energy-based mesh sampling and weighting for computational efficiency". International Journal for Numerical Methods in Engineering, 98(9), pp. 625-662.

[13] Farhat, C., Chapman, T., and Avery, P., 2015. "Structure-preserving, stability, and accuracy properties of the energyconserving sampling and weighting method for the hyper reduction of nonlinear finite element dynamic models". International Journal for Numerical Methods in Engineering, 102(5), pp. 1077-1110.

[14] Idelsohn, S. R., and Cardona, A., 1985. "A reduction method for nonlinear structural dynamic analysis". Computer Methods in Applied Mechanics and Engineering, 49(3), pp. 253 - 279.

[15] Rutzmoser, J., Rixen, D., Tiso, P., and Jain, S., 2017. "Generalization of quadratic manifolds for reduced order modeling of nonlinear structural dynamics". Computers \& Structures, 192, pp. $196-209$.

[16] Weeger, O., Wever, U., and Simeon, B., 2016. "On the use of modal derivatives for nonlinear model order reduction". International Journal for Numerical Methods in Engineering, 108(13), pp. 1579-1602.

[17] Jain, S., and Tiso, P., 2018. "Simulation-free hyper-reduction for geometrically nonlinear structural dynamics: A quadratic manifold lifting approach". Journal of Computational and Nonlinear Dynamics, 13(7), pp. 071003-07100312.

[18] Cappellini, N., Tamarozzi, T., Blockmans, B., Fiszer, J., Cosco, F., and Desmet, W., 2018. "Semi-analytic contact technique in a non-linear parametric model order reduction method for gear simulations". Meccanica, 53(1), Jan, pp. 49-75.

[19] Fiszer, J., Tamarozzi, T., Blockmans, B., and Desmet, W., 2015. "A time-dependent parametric model order reduction technique for modelling indirect bearing force measurements". Mechanism and Machine Theory, 83, pp. 152 - 174.

[20] Naets, F., De Gregoriis, D., and Desmet, W., 2019. "Multi-expansion modal reduction: A pragmatic semi-a priori model order reduction approach for nonlinear structural dynamics". International Journal for Numerical Methods in Engineering, 118(13), pp. 765-782.

[21] Tamarozzi, T., 2014. "Efficient numerical simulation strategies for flexible multibody systems with variable topology". $\mathrm{PhD}$ thesis, KU Leuven, Leuven, Belgium.

[22] Tamarozzi, T., Ziegler, P., Eberhard, P., and Desmet, W., 2013. "Static modes switching in gear contact simulation". Mechanism and Machine Theory, 63, pp. $89-106$.

[23] Ryckelynck, D., 2005. "A priori hyperreduction method: an adaptive approach". Journal of Computational Physics, 202(1), pp. $346-366$.

[24] Chaturantabut, S., and Sorensen, D. C., 2010. "Nonlinear model reduction via discrete empirical interpolation". SIAM Journal on Scientific Computing, 32(5), pp. 2737-2764.

[25] Tiso, P., and Rixen, D. J., 2013. "Discrete empirical interpolation method for finite element structural dynamics". In 
Topics in Nonlinear Dynamics, Volume 1, G. Kerschen, D. Adams, and A. Carrella, eds., Springer New York, pp. 203212.

[26] Rutzmoser, J., and Rixen, D., 2017. "A lean and efficient snapshot generation technique for the hyper-reduction of nonlinear structural dynamics". Computer Methods in Applied Mechanics and Engineering, 325, pp. 330 - 349.

[27] Vercammen, S., 2017. "Tyre/road noise due to road surface texture excitations: Experimental and numerical analyses". PhD thesis, KU Leuven, Leuven, Belgium.

[28] Nackenhorst, U., 2004. "The ALE-formulation of bodies in rolling contact: Theoretical foundations and finite element approach". Computer Methods in Applied Mechanics and Engineering, 193(39), pp. 4299-4322.

[29] Bathe, K.-J., 1996. Finite Element Procedures. Prentice Hall, Inc.

[30] Felippa, C., and Haugen, B., 2005. "A unified formulation of small-strain corotational finite elements: I. theory". Computer Methods in Applied Mechanics and Engineering, 194(21), pp. 2285 - 2335. Computational Methods for Shells.

[31] de Borst, R., Crisfield, M. A., Remmers, J. J., and Verhoosel, C. V., 2012. Non-linear Finite Element Analysis of Solids and Structures: Second Edition. John Wiley \& Sons, Ltd.

[32] Nackenhorst, U., 2014. "Finite element analysis of tires in rolling contact". GAMM-Mitteilungen, 37(1), pp. 27-65.

[33] Wriggers, P., 2006. Computational Contact Mechanics: Second Edition. Springer-Verlag.

[34] Ziefle, M., and Nackenhorst, U., 2008. "Numerical techniques for rolling rubber wheels: treatment of inelastic material properties and frictional contact”. Computational Mechanics, 42(3), Aug, pp. 337-356.

[35] Laursen, T. A., 2003. Computational Contact and Impact Mechanics. Springer-Verlag.

[36] Suwannachit, A., Nackenhorst, U., and Chiarello, R., 2012. "Stabilized numerical solution for transient dynamic contact of inelastic solids on rough surfaces". Computational Mechanics, 49(6), pp. 769-788.

[37] Zienkiewicz, O. C., Taylor, R. L., and Fox, D. D., 2013. The Finite Element Method for Solid and Structural Mechanics, 7th edition. Butterworth-Heineman.

[38] Arnold, M., and Brüls, O., 2007. "Convergence of the generalized- $\alpha$ scheme for constrained mechanical systems". Multibody System Dynamics, 18(2), pp. 185-202.

[39] Craig, R. R., and Kurdila, A. J., 2006. Fundamentals of structural dynamics, 2nd ed. ed. Wiley, Hoboken.

[40] Blockmans, B., 2018. "Model reduction of contact problems in flexible multibody dynamics". PhD thesis, KU Leuven, Leuven, Belgium.

[41] Helnwein, P., Liu, C., Meschke, G., and Mang, H., 1993. "A new 3-d finite element model for cord-reinforced rubber composites-application to analysis of automobile tires". Finite Elements in Analysis and Design, 14(1), pp. 1 - 16.

[42] Dassault Systèmes Simulia, 2012. Abaqus Example Problems Manual. Dassault Systèmes.

[43] Baranowski, P., Malachowski, J., Janiszewski, J., and Wekezer, J., 2016. "Detailed tyre fe modelling with multistage validation for dynamic analysis". Materials \& Design, 96, pp. 68 - 79.

[44] Guo, H., Bastien, C., Blundell, M., and Wood, G., 2014. "Development of a detailed aircraft tyre finite element model for safety assessment". Materials \& Design, 53, pp. $902-909$.

[45] Behroozi, M., Olatunbosun, O., and Ding, W., 2012. "Finite element analysis of aircraft tyre - effect of model complexity on tyre performance characteristics". Materials \& Design, 35, pp. 810 - 819. New Rubber Materials, Test Methods and Processes. 